\title{
Seção
}

\section{Relação de pareceristas ad hoc (2016-2017)}

Educação e Pesquisa agradece aos especialistas abaixo relacionados, que colaboraram com a revista no período de out. 2016 a nov. 2017.

Alcina Maria Testa Braz da Silva

Ada Ávila Assunção

Ademir Macedo Nascimento

Adir Casaro Nascimento

Adolfo Ignacio Calderón

Adrian Arroyo Pérez

Adriana Bauer

Adriana Bortoletto

Adriana Breda

Adriana Garcia Gonçalves

Adriana Marcondes Machado

Adriana Pugliese Netto Lamas

Agnaldo Arroio

Alan Augusto Moraes Ribeiro

Albertina Mitjans

Alberto Moreno Doña

Alda Junqueira Marin

Alessandra Rodrigues

Alexandre de Gusmão Pedrini

Alexandre Filordi de Carvalho

Alexandre Nascimento de Almeida

Alfredo José da Veiga-neto

Alice Pereira Xavier

Aline macedo Carvalho Freitas

Allan Moreira Xavier

Alvaro Moreira Hypolito

Amana Rocha Mattos

Amarilio Ferreira Neto

Américo Rodrigues de Almeida Neto

Ana Carina Stelko-Pereira

Ana Celuta Fulgêncio Taveira

Ana Claudia Balieiro Lodi

Ana Cristina Troncoso

Ana da Costa Polonia

Ana Elisa Spaolonzi Queiroz Assis

Ana Luísa Petersen Cogo

Ana Luiza B. Smolka

Ana Luiza Bustamante Smolka

Ana Maria Esteves Bortolanza

Ana Maria Fonseca de Almeida

Ana maria ortiz-colon

Ana Maria Villela Cavaliere

Ana Merces Bahia Bock
Ana Paula de Oliveira Corti

Ana Paula Soares da Silva

Ana Rebeca Miranda Castillo

Anderson Ferrari

Anderson Jonas das Neves

André Luis Corrêa

André Luis Tato

Andrea Cristina Filatro

Andréia Dalcin

Andreia de Bem Machado

Anete Abramowicz

Ângela Viana Machado Fernandes

Angelica Vier Munhoz

Antonia Terra Calazans Fernandes

Antonio Amorim

Antonio Carlos Rodrigues Amorim

Antonio Germano Magalhães Júnior

Antonio Hilario Aguilera Urquiza

Antonio Joaquim Severino

Aparecida Neri de Souza

Ariane Agnes Corradi

Artur José Renda Vitorino

Avelino Aldo de Lima Neto

Beatriz de Basto Teixeira

Belmira Amélia Oliveira Barros Bueno

Benerval Pinheiro Santos

Bernardete Angelina Gatti

Betania Ramalho

Bianca Cristina Correa

Bruno Cardoso de Menezes Bahia

Bruno Miguel de Almeida Dionísio

Camila Fernanda Bassetto Sampaio

Carla Biancha Angelucci

Carlos Augusto Candêo Fontanini

Carlos Eduardo Tabosa Lopes

Carlos Giovinazzo Junior

Carlos Herold Junior

Carlota Boto

Carmen Fernandez

Carolina de Roig Catini

Catarina Mangas

Cecilia Hanna Mate

Celso Garcia de Araújo Ramalho
Cely do Socorro Costa Nunes

Christiane Coutheux Trindade

Cintia Copit Freller

Cipriano Carlos Luckesi

Circe Maria Fernandes Bittencourt

Circe Mary Silva Silva,

Clarice Seixas Duarte

Clarilza Prado de Sousa

Clarissa Silva de Castilho

Claudemir Belintane

Claudia Dias Prioste

Claudia Lemos Vóvio

Cláudia Maria Mendes Gontijo

Cláudia Pereira Vianna

Claudia Regina Mosca Giroto

Cláudia Rosa Riolfi

Cláudio Antônio Tordino

Claudio Jose De Almeida Mello

Claudio Roberto Baptista

Cleoni Maria Barboza Fernandes

Cristiane Carneiro Capristano

Cristiane Coppe de Oliveira

Cristina Carvalho

Cristina Helena Almeida de Carvalho

Cristovam da Silva Alves

Cynthia Paes de Carvalho

Daniel Afonso da Silva

Daniel Fernando Bovolenta Ovigli,

Daniela Guerreiro-Casanova

Daniela Maria Ferreira

Daniervelin Renata Marques Pereira

Débora Goulart

Décio Gatti Júnior

Deise Mancebo

Denise Meyrelles de Jesus

Denise Silva Vilela

Diana de Souza Carvalho

Diana Vidal

Eder Coelho Paula

Eimard Gomes Antunes do Nascimento

Elenice de Souza Lodron Zuin

Elenilce Gomes de Oliveira

Eliana Albuquerque 
Eliane Gonçalves

Eliane Rose Maio

Elias Evangelista Gomes

Elie George Guimarães Ghanem Júnior

Élio Carlos Ricardo

Elisane Maria Rampelotto

Elizabeth Nogueira G.da Silva Mercuri

Elizabeth Santos Braga

Elizeu Clementino de Souza

Elvira Cristina Martins Tassoni

Emerson Freire

Enilda Rodrigues de Almeida Bueno

Ermelinda Moutinho Pataca

Evandro Alves

Fabiana Augusta Alves Jardim

Fabiana Soares Fernandes

Fábio de Godoy Del Picchia Zanoni

Fábio Hoffmann Pereira

Fabíola Menezes de Araujo

Fátima Elisabeth Denari

Fátima Niemeyer da Rocha

Fátima Suely Ribeiro Cunha

Fernando Bastos

Fernando Henrique Protetti

Fernando Luiz Cássio

Filomena Elaine Paiva Assolini

Flávia Faissal de Souza

Flávia Inês Schilling

Flavine Assis Miranda

Francisco B. Assumpção Jr.

Francisco José Figueiredo Coelho

Gabriela Miranda Moriconi

Gabriela Rodella Oliveira

Gamal Cerda Etchepare

Geisa do Socorro C V Mendes

Gilberto Francisco Dalmolin

Gilberto Icle

Gisela Maria do Val

Giselle Cristina Martins Real

Giselle Martins dos Santos Ferreira

Gizeli Fermino Coelho

Guilherme Castelo Branco

Heike Schmitz

Helena Maria Barroso Carvalho

Hustana Maria Vargas

Ione Ribeiro Valle

Isabel Cristina Moura Carvalho

Isabel Gretel María Eres Fernández

Isabel Melero Bello
Isabel Oliveira Silva

Itale Luciane Cericato

Jackson Gois da Silva

Jaime Francisco Parreira Cordeiro

Jair Lopes Junior

Jammilly Mikaela Fagundes Brandão

Jaqueline Batista de Oliveira Costa

Jerônimo Jorge Cavalcante Silva

João Sebastião

João Wanderley Geraldi

Jonatas Marcos da Silva Santos.

Jonathan Henriques do Amaral

Jorge Alarcón

José Armando Valente

José Augusto Brito Pacheco

José Carlos Souza Araujo

José Claudio Morelli Matos

José de Sousa Miguel Lopes

José Francisco Chicon

José Leon Crochick

José Luiz Zanella

José Manuel Resende

José Marcio Augusto de Oliveira

José Sérgio Fonseca de Carvalho

José Simões Almeida Jr.

Jucirema Quinteiro

Juliana Ap. M. Zechi

Juliana Aparecida Matias Zechi

Juliana Batista dos Reis

Juliana Zaganelli

Jussara Biagini

Katia Morosov Alonso

Katya Luciane Oliveira

Kimi Aparecida Tomizaki

Lana Mara de Castro Siman

Lea Pinheiro Paixão

Leda Maria de Oliveira Rodrigues

Leidy Gabriela Ariza

Leila Maria Ferreira Salles

Lélia Cristina Silveira de Moraes

Lenice Miranda Alves

Lenir Silva Abreu

Leonardo Leite de Andrade

Leonir Lorenzetti

Leonor L. Torres

Lidia Ruiz-Moreno

Lílian do Valle

Lisandra Ogg Gomes

Lubianka Ferrari Russo Rossi
Lucas Antônio Viana Botêlho

Lucia Leite

Lucia Pereira Leite

Luciana Garcia Mello

Luciana Massi

Luciane Marques Raupp

Lucídio Bianchetti

Luis Alexander Conde Solano

Luis Carlos Ribeiro Alves

Luís Gustavo Alexandre da Silva

Luis Marcelo Carvalho

Luiz Alberto Boing

Luiz Antonio Cunha

Luiz Augusto Coimbra Rezende Filho

Luiz Marcelo de Carvalho

Luiz Percival Leme Britto

Luz Elena Gallo

Magda Becker Soares

Manoel Oriosvaldo de Moura

Márcia Angela da Silva Aguiar,

Márcia Ondina Vieira Ferreira

Marcia Regina Oliveira Andrade

Márcia Zendron de Campos

Marco Antonio Leandro Barzano

Marcos Cezar de Freitas

Marcos Ferreira Santos

Marcos Garcia Neira

Marcos Namba Beccari

Marcos Traciso Masetto

Marcos Vinicius Francisco

Marcus Aurélio Taborda de Oliveira

Margaret Axt

Mari Valicheski Ferrari

Maria Amélia Rosário Santoro Franco

Maria Anória de Jesus Oliveira

Maria Aparecida Morgado

Maria Aparecida Viggiani Bicudo

Maria Caramez Carlotto

Maria Carla Corrochano

Maria Cecilia Sanches

Maria Ciavatta

Maria Cristina Cavaleiro

Maria Cristina Menezes

Maria das Graças Ch. de A. Nascimento

Maria de Fátima Barbosa Abdalla

Maria de Lourdes Haywanon S. Araujo

Maria de Lurdes Dias de Carvalho

Maria do Carmo de Lacerda Peixoto

Maria do Rosário Silveira Porto 
Maria do Socorro C. de Albuquerque

Maria Edgleuma de Andrade

Maria Elena Infante Malachias

Maria Elizabeth Bianconcini Trindade

Morato Pinto Almeida

Maria Fatima Felix Rosar

Maria Fátima Salum Moreira

Maria Goretti Queiroz

Maria Graça Jacintho Setton

Maria Isabel da Cunha

Maria Isabel da Silva Leme

Maria José Braga

Maria José Pinho

María Leonor Varas

Maria Letícia Barros Pedroso Nascimento

Maria Lourdes Rangel Tura

Maria Luísa Frazão Branco

Maria Luisa Merino Freitas Xavier

María Luisa Rodicio-García

Maria Lurdes Carvalho

Maria Márcia Sigrist Malavasi

Maria Osanette Medeiros

Maria Paula Parisi Lauria

Maria Regina Capelo

Maria Rita de Almeida Toledo

Maria Rosário Longo Mortatti

Maria Socorro Lucena Lima

Maria Teresa Vianna van Acker,

Maria Zélia Borba Rocha

Marieta Gouvêa de Oliveira Penna

Marilda Pasqual Schneider

Marília Pontes Sposito

Marina Jorge da Silva

Mário Henrique de Jesus Gomes

Marizete Lucini

Marli Elisa Dalmaso Afonso de André

Marli Lúcia Tonatto Zibetti

Marli Zibetti

Marlúcia Menezes de Paiva

Marta Maximo Pereira

Mauricio Ernica

Mauro Cavalcante Pequeno

Máximo Augusto Campos Masson

Máximo Daniel Lamela Adó

Michelle Prazeres Cunha

Miguel Luiz Ambrizzi

Milton Rosa

Miriam Cardoso Utsumi

Mirtes Cristina Marins de Oliveira
Mirtes Ribeiro Lira

Mitsuko Aparecida Makino Antunes

Mônica Cerbella Freire Mandarino

Mônica Dias Peregrino Ferreira

Monica Ribeiro Silva

Moysés Kuhlmann Júnior

Mylene Nogueira Teixeira

Nadir Zago

Narjara Mendes Garcia

Nathalia Cassettari

Neide Luzia de Rezende

Nelson Cardoso Amaral

Nerli Nonato Ribeiro Mori

Nesdete Mesquita Corrêa

Nicolás José Isola

Noeli Prestes Padilha Rivas

Norberto Dallabrida

Norma Sandra Almeida Ferreira

Núbio Delanne Ferraz Mafra

Nuria Hanglei Cacete

Odaci Luiz Coradini

Odair Sass

Oldimar Pontes Cardoso

Olgaises Cabral Maues

Patricia Margarida farias Coelho

Paula Arcoverde Cavalcante

Paula Leonardi

Paula Ramos de Oliveira

Paulo Alberto dos Santos Vieira

Paulo Henrique Arcas

Paulo Roberto Lima Jr

Paulo Rogério da Conceição Neves

Pedro Demo

Pedro Franco de Sá

Pedro Humberto Faria Campos

Pedro Rodrigues Magalhães Neto

Poliana Bruno Zuin

Rafaela Silva Rabelo

Regina Célia do Couto

Reinaldo Matias Fleuri

Renata Lopes Costa Prado

Renata Pereira Lima Aspis

Renato Cancian

Ricardo Desidério da Silva

Ricardo Ribeiro Terra

Rinaldo Voltolini

Rita Cássia Gallego

Roberto da Silva

Roberto José Montes Heloani
Roberto Paulo Machado Lopes

Roberto Rafael Dias da Silva

Rodrigo Manoel Dias da Silva

Rodrigo Pelegrini Ratier

Rodrigo Pelloso Gelamo

Rodrigo Petronio Ribeiro

Rodrigo Saballa de Carvalho

Romilda Teodora Ens

Ronaldo Barbosa

Ronaldo Marcos de Lima Araujo

Ronaldo Queiroz Morais

Ronice Quadros

Rosa Fátima Souza

Rosa Maria Bueno Fischer

Rosalia Duarte

Rosana Louro Ferreira Silva

Rosana Santos Jordão

Rosangela Aparecida Hilário

Rosangela Fritsch

Rosimeri de Oliveira Dias

Salomão Barros Ximenes

Sandoval Nonato

Sandra de Fátima Pereira Tosta

Sandra Eli Sartoreto de 0. Martins

Sandra Maria Sawaya

Sandra Maria Zákia Lian Sousa

Sauloéber Tarsio de Souza

Sebastião Jacinto dos Santos

Selma Borghi Venco

Selma Venco

Sibila Luft

Sidney Soares Filho

Silvana Pezzi

Silvia Helena Vieira Cruz

Silvia Kimo Costa

Sílvia Maria Agatti Lüdorf

Silvina Gvirtz

Silvio Donizetti de Oliveira Gallo

Simone Eliza do Carmo Lessa

Simone Magalhães Brito

Sinésio Ferraz Bueno

Sonia Carbonell Alvares

Sônia Cristina Vermelho

Sônia Maria Guedes Gondim

Sônia Maria Portella Kruppa

Sônia Teresinha Sousa Penin

Soraia Napoleão Freitas

Stela Mithá Duarte

Suerde Miranda de Oliveira Brito 
Susana Graciela Pérez Barrera Pérez Suzana dos Santos dos Santos Gomes Sydione Santos

Tania Chalhub

Tânia Cristina Rocha Silva Gusmão

Tânia Mara Cruz

Tânia Mara Pedroso Müller

Tarcísio de Arantes Leite

Thais Mere Marques Aveiro

Tiago Emanuel Klüber

Tizuko Morchida Kishimoto
Valderez Marina do Rosário Lima

Valéria Amorim Arantes

Vânia Lúcia Quintão Carneiro

Vera Lucia Gaspar da Silva

Vera Maria Nigro de Souza Placco

Vera Teresa Valdemarin

Victor Wolowski Kenski

Vinício de Macedo Santos

Vivian Batista da Silva

Vívian da Silva Lobato

Viviane Castro Camozzato

Viviani Alves de Lima
Wagner Bandeira Andriola

Walter Omar Kohan

Wanda Maria Junqueira de Aguiar

Wania Clemente Castro

Wendell Fiori de Faria

Wender Faleiro

Will Ribamar Mendes Almeida

Wilma de Nazaré Baía Coelho

Wilson José Alves Pedro

Wolney Gomes Almeida

Yara Araújo Ferreira Guimarães

Zaia Brandão

Zaqueu Vieira Oliveira 